\title{
Pluralismo plural en la concepción de Wittgenstein sobre seguir una regla*
}

\author{
Plural Pluralism in Wittgenstein's \\ view on rule-following
}

MANUEL PÉREZ OTERO**

\begin{abstract}
Resumen: Wittgenstein defiende una concepción pluralista sobre el seguimiento de reglas: es imposible que hubiera una única vez en que alguien siguiera una regla. Con frecuencia se le ha atribuido esa tesis porque es una consecuencia plausible de una concepción comunitarista que tal vez también propugna: es imposible que hubiera un único sujeto que siguiera alguna regla. En este artículo identifico una motivación diferente que Wittgenstein puede tener para sostener la concepción pluralista; es una motivación relacionada con la vaguedad de los procesos conducentes a adquirir la condición de seguidores de una regla.

Palabras clave: vaguedad / gradualismo / comunitarismo / costumbre / aprendizaje.
\end{abstract}

\begin{abstract}
Wittgenstein defends a pluralist view about rule-following: it is not possible that there should have been only one occasion on which someone followed a rule. Frequently, this thesis has been attributed to him because it is a plausible consequence of a communitarist view that he may also advocate: it is not possible that there should have been only one subject that followed a rule. In this paper I identify a different motivation that Wittgenstein can have for sustaining the pluralist view; a motivation related to the vagueness of the processes that lead to acquiring the condition of rule-followers.
\end{abstract}

Keywords: vagueness / gradualism / communitarianism / costum / learning.

Fecha de recepción: 29/05/2014. Fecha de aceptación: 21/10/2014.

* Una versión previa de este artículo se presentó en el II Seminario Wittgenstein, celebrado en Santiago de Chile en Otoño de 2011. Las ideas aquí expuestas se gestaron, principalmente, en un grupo de lectura LOGOS, dedicado al tema de seguir una regla, que coordiné en la Universidad de Barcelona durante el curso 2009-10. Agradezco a los participantes en esas reuniones sus comentarios y sugerencias, así como también a Mario Gómez-Torrente y Dan López de Sa, con quienes discutí algunos aspectos de sus contenidos. También estoy en deuda con dos evaluadores anónimos de Daimon. Financiación: Programa CONSOLIDER-INGENIO 2010, "Perspectival Thoughts and Facts" (CSD2009-00056), MICINN (gobierno de España). Proyecto "Conocimiento, referencia y realismo" (FFI2011-29560-C02-01), MICINN. Grupo de investigación consolidado LOGOS (2014SGR81), AGAUR (gobierno catalán).

** Universidad de Barcelona / LOGOS. Profesor Titular de Universidad. Email: perez.otero@ub.edu. Líneas de investigación: epistemología, filosofía del lenguaje, lógica filosófica, metafísica. Dos publicaciones recientes: "Purposes of Reasoning and (a New Vindication of) Moore's Proof of an External World", Synthese 190, 2013, pp. 4181-4200, DOI: 10.1007/s11229-013-0256-6; "Boghossian's Inference Argument against Content Externalism Reversed”, Philosophy and Phenomenological Research 89, 2014, pp. 159-181, DOI: 10.1111/j.1933-1592.2012.00613.x. 


\section{Pluralismo sobre el seguimiento de reglas}

El pluralismo a que hace referencia el primer sustantivo del título de este texto está contenido en una tesis mínima que se atribuye habitualmente al segundo Wittgenstein. Una posible formulación de dicha tesis pluralista débil sería la siguiente proposición, a la que voy a denominar Pluralismo (sobre Seguir una Regla):

(PSR): No es posible que una determinada regla, $\mathrm{R}$, sea seguida por un único sujeto y una única vez ${ }^{1}$.

Aunque este Pluralismo, PSR, constituye una tesis débil, es factible concebir alguna tesis pluralista más débil todavía (una tesis implicada por PSR pero que no implica PSR). Por ejemplo, dicha tesis pluralista más débil podría ser un Pluralismo Genérico (sobre Seguir una Regla) que estableciera esto:

(PGSR): No es posible que haya una única ocasión en que un único sujeto sigue alguna regla ${ }^{2}$.

Para cada regla $\mathrm{R}$, Pluralismo (PSR) establece un pluralismo respecto a $\mathrm{R}$ (un pluralismo específico respecto a $\mathrm{R}$, podríamos decir): no es posible seguirla en una única ocasión (y por un único sujeto). Pero la segunda tesis, Pluralismo Genérico (PGSR), establece meramente algo sobre la generalidad de las reglas: lo que no puede ocurrir es que sólo una regla sea seguida y sólo una vez (y por un único sujeto). Así, sería falso el Pluralismo pero verdadero el Pluralismo Genérico si cierta regla R se siguiera sólo en una ocasión pero otras reglas se siguieran también (y quizá, típicamente, en varias ocasiones).

Un parágrafo de las Investigaciones Filosóficas sugiere que Wittgenstein respalda la tesis comparativamente fuerte Pluralismo:

¿Es lo que llamamos "seguir una regla" algo que pudiera hacer sólo un hombre sólo una vez en la vida? [...] No puede haber sólo una única vez en que un hombre sigue una regla. [...] (Wittgenstein 1953, § 199).

Sin embargo, algún otro fragmento puede llevarnos a pensar que tal vez Wittgenstein se comprometería sólo con el Pluralismo Genérico. Por ejemplo, prestemos atención a esta cita:

Puedo tal vez, tal y como están las cosas, inventar un juego que nunca sea jugado por nadie._- ¿Pero sería también posible esto: La humanidad nunca ha jugado ningún juego; pero una vez alguien inventó un juego - que luego en verdad nunca fue jugado? (op. cit., § 204).

1 Por si algún lector quisiera atenerse a una versión más formalizada de Pluralismo, éste es otro modo en que podemos representar dicha tesis (PSR): No es posible [Hay un sujeto, S, una regla, R, y una ocasión, t, tales que: $S$ sigue la regla $R$ en $t$ y (si un sujeto, $S^{*}$, sigue la regla $R$ en la ocasión $t^{*}$, entonces $S=S^{*} y t=t^{*}$ )].

2 He aquí una versión semi-formalizada de ese Pluralismo Genérico (PGSR): No es posible [Hay un sujeto, S, una regla, $\mathrm{R}$, y una ocasión, $\mathrm{t}$, tales que: $\mathrm{S}$ sigue la regla $\mathrm{R}$ en $\mathrm{t}$ y (si un sujeto, $\mathrm{S}$, sigue la regla $\mathrm{R}^{*}$ en la ocasión $\mathrm{t}^{*}$, entonces $\left.\left.\mathrm{S}=\mathrm{S}^{*}, \mathrm{R}=\mathrm{R}^{*} \mathrm{yt}=\mathrm{t}^{*}\right)\right]$. 
Para poner en relación ese fragmento con la cuestión de seguir una regla, conviene tener en cuenta que aunque Wittgenstein considerase que algunos juegos no están regidos por reglas, nada hace suponer que la reflexión que está proponiendo deba restringirse a ese tipo de juegos. Supongamos, entonces, que dicha reflexión se aplica también - según Wittgenstein - a aquellos juegos regidos por reglas (en los cuales jugar comporta seguir alguna regla). Eso no basta para interpretar el fragmento en un sentido favorable al Pluralismo Genérico y quizá compatible con la negación del Pluralismo. Haría falta suponer también que ese juego que tal vez puede inventarse y no ser jugado nunca, podría alternativamente inventarse y ser jugado una única vez (contrariamente al Pluralismo). Por otra parte, reinterpretando análogamente la pregunta de la cita, tendríamos este resultado: ¿sería posible esto: la humanidad nunca ha jugado ningún juego; pero una vez alguien inventó un juego - que luego fue jugado en una única ocasión? Si aceptamos que Wittgenstein también contestaría negativamente a esta pregunta (como sugiere que debe contestarse su pregunta original), obtenemos su compromiso con la tesis débil del Pluralismo Genérico.

Sostener la tesis Pluralismo Genérico sin comprometerse con Pluralismo podría estar motivado por la creencia de que aunque pueda haber - excepcionalmente - un caso único de seguir cierta regla $\mathrm{R}$, ese seguimiento deriva de otros casos de seguir una regla, si bien estos son casos en que la regla seguida no necesariamente es R, sino alguna otra regla (típicamente, algunas otras reglas).

\section{Comunitarismo}

El tema principal que quiero resaltar no es el contraste entre el Pluralismo y su consecuencia Pluralismo Genérico, sino el contraste entre cualquiera de ellas y tesis pluralistas más fuertes. Una versión más comprometida del pluralismo es el comunitarismo (sobre seguir una regla), que también ha sido atribuido usualmente a Wittgenstein, aunque dicha atribución no resulta tan trivial. Una formulación del Comunitarismo (sobre Seguir una Regla) sería CSR:

(CSR): No es posible que una determinada regla, $\mathrm{R}$, sea seguida por un único sujeto ${ }^{3}$.

Según el Comunitarismo, algunas de las otras ocasiones en que una regla es seguida serán casos en que otros sujetos la siguen. El Comunitarismo implica el Pluralismo (y por tanto implica el Pluralismo Genérico), pero la inversa no es cierta.

Existiría también una versión genérica del Comunitarismo: una tesis que se relaciona con el Comunitarismo como el Pluralismo Genérico se relaciona con el Pluralismo. Ese Comunitarismo Genérico (sobre Seguir una Regla) rezaría así:

(CGSR): No es posible que un sujeto sea el único que sigue alguna regla ${ }^{4}$.

3 Ésta es una versión semi-formal del Comunitarismo (CSR): No es posible [Hay un sujeto, S, y una regla, R, tales que: $\mathrm{S}$ sigue la regla $\mathrm{R}$ y ( si un sujeto, $\mathrm{S}^{*}$, sigue la regla $\mathrm{R}$, entonces $\mathrm{S}=\mathrm{S} *$ )].

4 La versión semi-formal del Comunitarismo Genérico (CGSR) sería la siguiente: No es posible [Hay un sujeto, $\mathrm{S}$, y una regla, $\mathrm{R}$, tales que: $\mathrm{S}$ sigue la regla $\mathrm{R}$ y ( $\mathrm{si}$ un sujeto, $\mathrm{S}^{*}$, sigue la regla $\mathrm{R}^{*}$, entonces $\mathrm{S}=\mathrm{S} *$ )]. 
El Comunitarismo implica el Comunitarismo Genérico pero no viceversa. El Comunitarismo Genérico implica el Pluralismo Genérico pero no viceversa. Pero tampoco será tema prominente aquí el contraste entre Comunitarismo y Comunitarismo Genérico.

\section{Pluralismo basado en la vaguedad}

Mi tema principal en este artículo concierne al adjetivo, plural, contenido en el título. Mediante él pretendo referirme a la pluralidad de motivaciones presentes en el segundo Wittgenstein para sostener una posición al menos débilmente pluralista en el sentido del Pluralismo o del Pluralismo Genérico. De una de esas motivaciones se ha escrito mucho, especialmente desde la publicación de Kripke (1982); procede precisamente del Comunitarismo o del Comunitarismo Genérico. Según esa interpretación kripkeana, Wittgenstein sostendría al menos el Pluralismo, o el Pluralismo Genérico, basándose en consideraciones comunitaristas, que le comprometen por tanto también con alguna de las tesis más fuertes, el Comunitarismo o el Comunitarismo Genérico. Mi tesis principal es que existen motivaciones en Wittgenstein para sostener el Pluralismo o el Pluralismo Genérico con independencia (al menos aparentemente) de tales consideraciones comunitaristas.

Justamente porque propongo una pluralidad de motivaciones wittgensteinianas para sostener el Pluralismo o el Pluralismo Genérico no niego que sea correcto atribuir a Wittgenstein también el Comunitarismo o el Comunitarismo Genérico. Ni siquiera niego que las razones principales de su pluralismo (sobre seguir una regla) estén contenidas en ese comunitarismo. Ésa es una controversia que no abordaré. Afirmo, no obstante, que hay también al menos otra motivación. Sostengo también que es aceptable asumir que convivieran en Wittgenstein esas diversas tesis (fundamentadas en consideraciones independientes entre sí) que implican, cada una de ellas, el Pluralismo o el Pluralismo Genérico. Ese pluralismo de motivaciones, por otra parte, está plenamente en consonancia tanto con el estilo de los textos del Wittgenstein de esa época como con sus posiciones metodológicas.

Las otras razones a las que me refiero proceden, principalmente, de la vaguedad, o la gradualidad, del proceso por el cual un sujeto adquiere la condición de ser alguien que puede seguir una determinada regla. En general, la vaguedad, o la gradualidad, respecto a cualquier fenómeno claramente parece implicar pluralidad. Por ejemplo, la vaguedad (real o potencial) involucrada en ser alto, aplicado a personas, implica que hay una pluralidad de alturas que las personas tienen (o pueden tener). Si todos midiésemos 1'80 cm. no habría vaguedad respecto a esa propiedad. Nuestras intuiciones pre-teóricas respaldan también este juicio más concreto: no hay una altura mínima $m$, tal que un sujeto es alto si y sólo si mide al menos $m \mathrm{~cm}^{5}$.

5 Diferentes teóricos sobre la vaguedad (epistemicistas, supervaluacionistas y otros; cf., por ejemplo, Williamson 1994 y Fine 1975) negarían ese juicio. Muy probablemente, Wittgenstein rechazaría tales posturas (que podríamos clasificar como) negacionistas respecto a la vaguedad. Yo me inclino a creer que el negacionismo es erróneo. Sería deseable, sin embargo, que mi análisis no dependiera de ello. Creo que las teorías negacionistas también postulan cierta pluralidad como la que estoy señalando, derivada de la vaguedad, de modo que, en lo sustancial, podrían reformularse mis reflexiones sin prejuzgar nada contra esas teorías. Pero, por simplicidad, continuaré con las formulaciones menos neutras. Además, en sentido estricto mi propuesta no depende del rechazo del negacionismo, pues mi propuesta es principalmente una interpretación de Wittgenstein. (Estoy en deuda con Mario Gómez-Torrente y con Dan López de Sa por sus comentarios en relación con estos asuntos.) 
Veamos un caso más similar al seguimiento de reglas: para cualquier tipo de acción $\mathrm{F}$, tener el hábito o la costumbre de (hacer) $F$ es un tipo de acción que involucra cierta gradualidad. Aquí 'hábito' y 'costumbre' admiten ser interpretados en un sentido meramente estadístico, por decirlo así, que no implica ningún aspecto social o comunitario. S tiene la costumbre de (hacer) F sólo implicaría (conforme a esa lectura meramente estadística) que $\mathrm{S}$ suele (hacer) F. La tesis clave sobre tales hábitos (análoga a la tesis sobre la altura) sería ésta: no existe un primer caso de soler (hacer) $\mathrm{F}$, incluso aunque exista un primer caso de (hacer) F. Es decir, no existe una primera ocasión, t, tal que $\mathrm{S}$ hace $\mathrm{F}$ en $\mathrm{t}$ y adquiere en $\mathrm{t}$ la condición de tener la costumbre de hacer F. ${ }^{6}$

Wittgenstein identifica seguir una regla con una costumbre. También señala, respecto a ciertos casos de seguir una regla, que no existe una primera ocasión en que se sigue la regla. En relación con lo primero, son relevantes fragmentos como éste:

[...] Seguir una regla, hacer un informe, dar una orden, jugar una partida de ajedrez son costumbres (usos, instituciones) [...] (op. cit., § 199).

Así, según Wittgenstein seguir una regla sería actuar conforme a una costumbre. (No deduzcamos de ello que, inversamente, todo caso de actuar conforme a una costumbre sea un caso de seguir una regla.) Cabe suponer que Wittgenstein está apelando ahí a aspectos sociales del concepto de costumbre (que mencione también instituciones apoya esa idea). Eso apuntaría a tesis comunitaristas como el Comunitarismo o el Comunitarismo Genérico. El punto clave que quiero destacar es el siguiente: aunque Wittgenstein sólo invocara un sentido más neutro (y más débil) de 'costumbre' (el sentido meramente estadístico al que me refería), eso bastaría para sostener el pluralismo mínimo de Pluralismo o de Pluralismo Genérico. ${ }^{7}$

La vaguedad o gradualidad en el seguimiento de reglas conllevaría una tesis, Gradualismo (sobre Seguir una Regla), que podría formularse así:

(GSR): No es posible que exista una primera vez en que alguien sigue una determinada regla, $\mathrm{R} .^{8}$

6 Las construcciones 'tiene la costumbre de hacer F' y 'suele (hacer) F' frecuentemente van acompañadas de algún modificador, del tipo 'en (las circunstancias) $Q$ ' (en verano, cuando era niño, los fines de semana, si voy a Madrid, por la noche, cuando su padre le mira, etc.). Por ello, la gradualidad puede proceder también de otra fuente: puede no haber tampoco un primer verano a partir del cual S adquiriera la condición de tener la costumbre de ir a la playa en verano. Pero pudo haber un primer verano con esa propiedad. En cualquier caso - conforme a la tesis indicada en el texto principal - no hubo una primera ocasión $\mathrm{t}$, tal que $\mathrm{S}$ fue a la playa en t y adquirió en t la condición de tener la costumbre de ir a la playa en verano.

7 En cualquier caso, no cabe identificar estos dos conceptos: S actúa conforme a su costumbre de hacer R (que implica que S tiene la costumbre de hacer R) / S actúa conforme a la costumbre de hacer R (que implica que existe la costumbre de hacer R). Por otra parte, aunque la costumbre en cuestión sea una regla, ese contraste no debe confundirse con el contraste entre Pluralismo y Pluralismo Genérico ni con el contraste entre Comunitarismo y Comunitarismo Genérico.

8 Una versión semi-formal de este Gradualismo (GSR): No es posible [Hay un sujeto, S, una regla, R, y una ocasión, $t$, tales que: $\mathrm{S}$ sigue la regla $\mathrm{R}$ en $\mathrm{t}$ y (si un sujeto, $\mathrm{S}^{*}$, sigue la regla $\mathrm{R}$ en la ocasión $\mathrm{t}^{*}$, entonces $\mathrm{t}^{*}$ no es anterior a $\mathrm{t}$ )]. 
Existiría también una versión genérica de ese gradualismo. Este Gradualismo Genérico (sobre Seguir una Regla) establecería:

(GGSR): No es posible que exista una primera vez en que alguien sigue alguna regla ${ }^{9}$.

El Gradualismo y el Gradualismo Genérico mantienen entre sí y con, respectivamente, el Pluralismo y el Pluralismo Genérico relaciones análogas a las del Comunitarismo y el Comunitarismo Genérico. Es decir: el Gradualismo implica el Gradualismo Genérico pero no viceversa. El Gradualismo implica el Pluralismo pero no viceversa. El Gradualismo Genérico implica el Pluralismo Genérico pero no viceversa.

Tal y como he sostenido, el Gradualismo y el Gradualismo Genérico proporcionan motivaciones para el pluralismo (para el Pluralismo o para el Pluralismo Genérico) aparentemente independientes del Comunitarismo (sea CSR o CGSR). Creo que, aparentemente, el Gradualismo y el Gradualismo Genérico son tesis correctas. De todos modos, en la próxima sección aporto indicios adicionales sobre por qué el Gradualismo y el Gradualismo Genérico podrían ser verdaderas.

Algunos lectores tal vez se pregunten qué significa que un concepto sea vago ${ }^{10}$. Es esa una interrogación que podría abordarse con diferentes niveles de detalle. Simplificando mucho, habría al menos dos estrategias de respuesta posibles: (a) ilustrar la noción con algunos casos suficientemente representativos de lo que se quiere decir al hablar aquí de vaguedad; (b) hacer eso mismo, pero reforzar la respuesta con una descripción adicional de lo que las principales teorías sobre ese fenómeno (la vaguedad) sostienen, indicando cuál me parece preferible y por qué. Voy a seguir solo la estrategia (a), porque considero que los objetivos de este trabajo no requieren seguir la segunda estrategia, si bien también haré algunos comentarios (adicionales a lo indicado en la nota 5) en el sentido de esa estrategia (b). Conceptos paradigmáticamente vagos serían, por ejemplo, los siguientes: alto, lejano, importante, calvo, simpático, joven. Y la vaguedad en cuestión está íntimamente conectada con el siguiente hecho (si es que no consiste precisamente en eso): para cada uno de esos conceptos, podemos concebir una pluralidad de items a los cuales se aplica el concepto en diferentes grados, de tal modo que para algún item no parece estar determinado si el concepto se le aplica o no. Mi tesis principal en este artículo (la vaguedad presente en los procesos cuyo resultado es tener la condición de poder seguir una regla proporciona a Wittgenstein una motivación favorable al pluralismo, incluso si Wittgenstein no hubiera sido comunitarista) depende de esta otra tesis: el concepto de seguir una regla también parece ser un concepto vago. Su vaguedad se pondría de manifiesto (como he indicado) en que las tesis Gradualismo y Gradualismo Genérico resultan muy plausibles (cf. también la próxima sección).

9 La versión semi-formal del Gradualismo Genérico (GGSR): No es posible [Hay un sujeto, S, una regla, R, y una ocasión, $t$, tales que: $\mathrm{S}$ sigue la regla $\mathrm{R}$ en $\mathrm{t}$ y (si un sujeto, $\mathrm{S}^{*}$, sigue la regla $\mathrm{R} *$ en la ocasión $\mathrm{t}^{*}$, entonces $\mathrm{t}^{*}$ no es anterior a $\mathrm{t}$ )].

10 Las consideraciones en este párrafo y en el siguiente vienen motivadas por diversas cuestiones planteadas por un anónimo evaluador de Daimon. 
En relación con la estrategia (b), en la literatura filosófica contemporánea encontramos teorizaciones sobre la vaguedad muy diferentes entre sí. Tal vez el supervaluacionismo sea la teoría menos problemática de entre todas esas propuestas (cf., por ejemplo, Fine 1975 o Varzi 2001). Pero tiendo a simpatizar con quienes consideran (no me refiero solo a Wittgenstein) que en este terreno la teoría menos mala probablemente no sea suficientemente buena.

\section{Consideraciones en favor del gradualismo: gramática y aprendizaje}

¿Qué motivaciones no comunitaristas podría tener Wittgenstein en favor de ese pluralismo representado por el Gradualismo y el Gradualismo Genérico? Voy a distinguir dos razones, o dos tipos de razones.

Existiría una posible razón meramente lógico-conceptual (gramatical, en terminología de Wittgenstein). Podría sostenerse que 'seguir la regla $\mathrm{R}$ ' implica de forma no obvia algo similar (el pluralismo) a lo que 'tener la costumbre de hacer R' o 'soler hacer R' implican de forma manifiesta. La implicación sería un rasgo o característica lógico-gramatical de dicha expresión. La segunda parte de esta cita iría en ese sentido (en la continuación de ese parágrafo Wittgenstein da una respuesta negativa a la pregunta planteada):

¿Es lo que llamamos "seguir una regla” algo que pudiera hacer sólo un hombre sólo una vez en la vida? - Y ésta es naturalmente una anotación sobre la gramática de la expresión "seguir una regla". [...] (op. cit., § 199).

Conforme a esa primera razón, lógico-conceptual-gramatical, lo que sucedería - repitámoslo- es que la expresión lingüística 'seguir la regla R' compartiría con 'tener la costumbre de hacer R' o con 'soler hacer R' un rasgo lógico-gramatical (aunque su posesión de ese rasgo sería menos obvia): el rasgo que implica (en relación a esas otras dos expresiones) que es imposible tener la costumbre de hacer $\mathrm{R}$ o soler hacer $\mathrm{R}$ mediante un único acto.

Quiero referirme también a otra razón o motivación, más específica. Concierne a los procesos de aprendizaje de reglas. Wittgenstein la menciona en relación con ciertos casos de seguir una regla: los que involucran tareas de "lectura" (en un sentido concreto de 'leer', que no implica la comprensión del sentido de lo leído, sino una actividad bajo la cual se incluyen transformar en sonidos lo escrito o impreso, escribir al dictado, transcribir lo impreso, tocar siguiendo una partitura y otras cosas por el estilo; cf. op. cit. $\$ 156)$.

Cuando un sujeto, $\mathrm{S}$ (un niño, por ejemplo), hace pruebas de tanteo (quizá instruido por un experto) hasta que puede comprobarse que ha captado y aplica correctamente determinada regla no hay una línea divisoria clara — al menos según la opinión de Wittgenstein; cf. mi nota 5 - entre los casos en que $\mathrm{S}$ todavía no seguía la regla y los casos en que $\mathrm{S}$ ya sigue la regla. Ejemplificado con casos de leer:

Considera el siguiente caso: Seres humanos, u otros seres, son utilizados por nosotros como máquinas de leer. Son adiestrados para este fin. Quien los adiestra dice de uno que ya puede leer y de otros que aún no pueden. Toma el caso de un alumno que hasta ahora no ha participado: si se le muestra una palabra escrita producirá a veces 
algún tipo de sonido y aquí y allá sucede 'casualmente' que es más o menos exacto. Una tercera persona oye a este alumno en un caso así y dice: 'Él lee'. Pero el maestro dice: "No, no lee; fue sólo una casualidad."-Pero supongamos que este alumno cuando se le presentan otras palabras, continúa reaccionando a ellas correctamente. Después de un tiempo el maestro dice: “¡Ahora puede leer!”-¿Pero qué pasó con aquella primera palabra? ¿Debe el maestro decir: "Me había equivocado, él leía $a$ pesar de todo"? - o: "Él sólo ha comenzado a leer realmente después"? — ¿Cuándo ha comenzado a leer? ¿Cuál es la primera palabra que ha leído? Esta pregunta es aquí carente de sentido. [...] (op. cit., § 157. Cf. también Wittgenstein 1958, p. 159).

[...] hay una serie continua de transiciones entre el caso en que alguien recita de memoria lo que debe leer y aquél en que lee cada palabra letra por letra sin ayudarse de la conjetura por el contexto ni del saber de memoria. [...] (Wittgenstein 1953, § 161. Cf. también Wittgenstein 1958, p. 161).

Otros pasajes aluden a casos similares (pero no equivalentes) en que también es gradual la diferencia entre seguir correctamente una regla y no hacerlo. Así, por ejemplo:

[...] Pero supongamos ahora que, tras algunos esfuerzos del maestro, él continúa la serie correctamente, es decir, como lo hacemos nosotros. Pues bien, ahora podemos decir: él domina el sistema._¿Pero hasta dónde tiene él que continuar la serie correctamente para que tengamos derecho a decirlo? Está claro: no puedes indicar aquí límite alguno. [...] (Wittgenstein 1953, § 145).

Hago explícita la conexión entre estos datos concretos textuales y la tesis general de este artículo. Esa tesis general es que la vaguedad presente en los procesos cuyo resultado es tener la condición de poder seguir una regla proporcionan a Wittgenstein una motivación favorable al pluralismo, incluso si Witgenstein no hubiera sido comunitarista. La tesis se vería apoyada por datos favorables a las tesis pluralistas precisas Gradualismo y Gradualismo Genérico. Pues bien, las consideraciones de Wittgenstein en estos pasajes indican que la reflexión atenta sobre los procesos de aprendizaje de reglas apoya las tesis pluralistas Gradualismo y Gradualismo Genérico, basadas en el carácter gradual de los procesos que conducen a poder seguir una regla (incluyendo tales procesos de aprendizaje). Y, más concretamente, dada la naturaleza de cada proceso individual de aprendizaje, sería muy poco plausible suponer que Wittgenstein aceptaría sólo la tesis débil Gradualismo Genérico y contemplara la posibilidad de casos incompatibles con el Gradualismo ${ }^{11}$. En definitiva, creo haber aportado razones de peso para sostener mi tesis principal.

11 Fogelin (1976-1987) ha defendido que el proceso de aprendizaje de reglas debe ser comunitario. Si fuera así, el pluralismo derivado de la gradualidad de tales aprendizajes no sería independiente del comunitarismo. Pero creo que esa tesis de Fogelin es muy controvertida y me parece insuficiente la justificación que ofrece en su favor.

Por otra parte, algunos autores (cf., por ejemplo, Schulte 2010, pp. 32-33) rechazan que los pasajes de Wittgenstein sobre leer anticipen sus reflexiones sobre seguir una regla. No obstante, incluso si esos pasajes no anticipa- 


\section{Bibliografía}

Fine, Kit (1975): "Vagueness, Truth and Logic", Synthese 30, pp. 265-300.

Fogelin, Robert J. (1976-1987): Wittgenstein, London: Routledge, 1976. Segunda edición (ampliada): 1987.

Kripke, Saul (1982): Wittgenstein on Rules and Private Languages. An Elementary Exposition, Cambridge: Harvard University Press, 1982. Traducciones: Wittgenstein a propósito de reglas y lenguaje privado, Madrid: Tecnos, 2006. Reglas y lenguaje privado en Wittgenstein, México: UNAM, 1989.

Schulte, Joachim (2010): "Reading-machines, Feelings of Influence, Experiences of Being Guided: Wittgenstein on Reading”, en Pasquale Frascolla, Diego Marconi y Alberto Voltolini (eds.), Wittgenstein: Minds, Meaning and Metaphilosophy, Basingstoke: Palgrave Macmillan, pp. 31-45.

Varzi, Achille C. (2001): "Vagueness, logic and ontology", The Dialogue. Yearbooks for Philosophical Hermeneutics 1, pp. 135-154.

Williamson, Timothy (1994): Vagueness, London: Routledge.

Wittgenstein, Ludwig (1953): Investigaciones filosóficas. Barcelona: Crítica, 1988. Edición bilingüe. Original: Philosophische Untersuchungen (publicado en 1953).

Wittgenstein, Ludwig (1958): Los cuadernos azul y marrón, Madrid: Tecnos, 1968. Original: The Blue and Brown Books, Oxford: Basil Blackwell, 1958.

ran el problema relativo a seguir una regla, Wittgenstein considera que leer es un caso de seguir una regla. Eso es suficiente para mis propósitos. 
